Note

\title{
Scattering by randomly oriented thin ice disks with moderate equivalent-sphere size parameters
}

\author{
Nadia T. Zakharova ${ }^{\mathrm{a}, *}$ and Michael I. Mishchenko ${ }^{\mathrm{b}}$ \\ ${ }^{a}$ SGT, Inc., 2880 Broadway, New York, NY 10025, USA \\ ${ }^{\mathrm{b}}$ NASA Goddard Institute for Space Studies, 2880 Broadway, New York, NY 10025, USA
}

\begin{abstract}
We use the $T$-matrix method to compute the scattering matrix for randomly oriented circular ice cylinders with diameter-to-length ratios 1 and 20 and surface-equivalent-sphere size parameters up to 12 . We show that wavelength-sized, sharp-edged ice plates with extreme diameter-to-length ratios possess the same scattering properties as smooth platelike spheroids: their phase functions are similar to those of surface-equivalent compact particles, whereas all other elements of the scattering matrix are typical of Rayleigh scattering.
\end{abstract}

Keywords: Electromagnetic scattering; Nonspherical particles; $T$-matrix method; Remote sensing

*Corresponding author. Tel.: +1-212-678-5590; fax: +1-212-678-5552.

E-mail address: crntz@giss.nasa.gov (N.T. Zakharova). 
In a recent paper [1], we have applied the $T$-matrix method [2,3] to compute light scattering by randomly oriented prolate and oblate ice spheroids with aspect ratios up to 20 and surfaceequivalent-sphere size parameters up to 12 . (The aspect ratio is defined as the ratio of the largest to the smallest particle dimensions.) We have demonstrated that plate-like and needle-like ice spheroids with moderate size parameters possess unique scattering properties: their phase functions are similar to those of surface-equivalent compact particles, whereas all other elements of the scattering matrix are typical of Rayleigh scatterers. Because the value of the size parameter along the shorter axis of the highly aspherical spheroids studied was less than 1 , we have concluded that the phase function for such particles is mostly determined by the value of the size parameter of the sphere having the same surface area, whereas all other elements of the scattering matrix are sensitive to the value of the size parameter along the smallest particle dimension (see also $[4,5]$ ).

Since spheroids are particles with smooth surfaces, it is important to verify whether sharpedged wavelength-sized particles with extreme aspect ratios possess similar scattering properties. The simplest type of sharp-edged particles that can be studied with the $T$-matrix code described in [6] is a finite circular cylinder [7]. The computation of light scattering by prolate ice cylinders with very large length-to-diameter ratios turned out to be problematic because of convergence problems discussed in [6]. However, we have been able to perform computations for randomly oriented oblate ice cylinders with equal-surface-sphere size parameters up to 12 and diameter-tolength ratios as large as 20 .

The results of our computations are summarized in Figs. 1-3, which depict the phase function $a_{1}(\Theta)$ and the normalized elements of the Stokes scattering matrix [8]

$$
\mathbf{F}(\Theta)=\left[\begin{array}{cccc}
a_{1}(\Theta) & b_{1}(\Theta) & 0 & 0 \\
b_{1}(\Theta) & a_{2}(\Theta) & 0 & 0 \\
0 & 0 & a_{3}(\Theta) & b_{2}(\Theta) \\
0 & 0 & -b_{2}(\Theta) & a_{4}(\Theta)
\end{array}\right]
$$

where $\Theta$ is scattering angle. As usual, the phase function satisfies the normalization condition

$$
\frac{1}{2} \int_{0}^{\pi} \mathrm{d} \Theta \sin \Theta a_{1}(\Theta)=1
$$

We show results for cylinders with diameter-to-length ratios $D / L=1$ and 20 and for surfaceequivalent spheres. As in [1], we have suppressed the interference structure by averaging the 
results for spheres and cylinders with $D / L=1$ over a narrow gamma distribution of surfaceequivalent-sphere radii $r_{\mathrm{s}}$ given by [9]

$$
n\left(r_{\mathrm{s}}\right)=\frac{1}{r_{\mathrm{s}, \mathrm{eff}} v_{\text {eff }} \Gamma\left(\frac{1-2 v_{\text {eff }}}{v_{\text {eff }}}\right)}\left(\frac{r_{\mathrm{s}}}{r_{\mathrm{s}, \text { eff }} v_{\text {eff }}}\right)^{\left(1-3 v_{\text {eff }}\right) / v_{\text {eff }}} \exp \left(-\frac{r_{\mathrm{s}}}{r_{\mathrm{s}, \mathrm{eff}} v_{\text {eff }}}\right)
$$

with an effective variance of $v_{\text {eff }}=0.05$. The curves for monodisperse cylinders with $D / L=20$ were sufficiently smooth and did not require averaging over sizes. Accordingly, the size of cylinders with $D / L=20$ is specified in Figs. $1-3$ in terms of the monodisperse surfaceequivalent-sphere size parameter $x_{\mathrm{s}}=2 \pi r_{\mathrm{s}} / \lambda$, whereas the size of polydisperse spheres and cylinders with $D / L=1$ is specified in terms of the effective surface-equivalent-sphere size parameter $x_{\mathrm{s}, \text { eff }}=2 \pi r_{\mathrm{s}, \mathrm{eff}} / \lambda$, where $\lambda$ is the wavelength of the incident light. The refractive index is 1.311 .

Examination of Figs. 1-3 and figures 2 and 3 from [1] shows that despite their sharp-edged shapes, wavelength-sized circular ice cylinders with extreme aspect ratios possess the same scattering properties as smooth platelike spheroids. Specifically, their phase functions are similar to those of surface-equivalent spheres and nonspherical particles (spheroids and cylinders) with moderate aspect ratios and have a forward-scattering lobe with magnitude rapidly increasing with size parameter. In contrast, all other elements of the scattering matrix closely resemble those of the Rayleigh scattering matrix as long as the size parameter along the smallest cylinder dimension is less than 1. For example, all curves of linear polarization $\left(-b_{1} / a_{1}\right)$ for plates with $D / L=20$ have the renowned bell-like shape with a maximum reaching almost $100 \%$ at side-scattering angles. Unlike the case for compact particles, the scattering angle of maximal positive polarization decreases rather than increases with increasing size parameter. The ratio $a_{2}(\Theta) / a_{1}(\Theta)$ is close to unity, the elements $a_{3}(\Theta)$ and $a_{4}(\Theta)$ are almost the same and do not vary significantly with size parameter, and the ratio $b_{2}(\Theta) / a_{1}(\Theta)$ is close to zero at most scattering angles. This behavior differs very much from that exhibited by surface-equivalent spheres and compact nonspherical particles.

Our theoretical computations are in excellent agreement with the results of recent laboratory measurements of electromagnetic scattering by plates with very large width-to-thickness ratios 
and thicknesses smaller than a wavelength [10]. Indeed, these microwave analog measurements also show phase functions characteristic of compact wavelength-sized scatterers and polarization curves typical of Rayleigh scattering. In particular, the observed maximal polarization values reach values close to $100 \%$ and occur at scattering angles less than $90^{\circ}$.

As discussed in [1], unusual scattering properties of wavelength-sized particles with extreme aspect ratios should be accounted for in analyses of laboratory and remote-sensing measurements of light scattering. For example, the fact that $a_{2}(\pi) \approx a_{1}(\pi)$ and $a_{4}(\pi) \approx-a_{1}(\pi)$ for needlelike and platelike particles with moderate size parameters makes the linear and circular depolarization ratios $[11,12]$ close to zero. Therefore, small measured values of depolarization should not be automatically interpreted as an indication of Rayleigh scattering or the spherical particle shape. Similarly, measurements of Rayleigh-like polarization (e.g. [13-16]) should not be automatically attributed to particles much smaller than a wavelength.

The simplicity of the scattering matrix for needlelike and platelike particles with moderate size parameters allows a convenient analytical parameterization similar to those developed in $[17,18]$. Such parameterizations can be very useful in first-order analyses of remote sensing observations when the plausible range of particle microphysical characteristics is unknown and is difficult to estimate a priori. Also, our results provide a benchmark for checking the accuracy of approximate formulations of light scattering by wavelength-sized particles with one dimension much smaller than the wavelength [19-22].

This research was sponsored by the NASA Radiation Science Program managed by Donald Anderson.

\section{References}

[1] Zakharova NT, Mishchenko MI. Scattering properties of needlelike and platelike ice spheroids with moderate size parameters. Appl Opt 2000; 39: 5052-5057.

[2] Mishchenko MI, Travis LD, Mackowski DW. T-matrix computations of light scattering by nonspherical particles: a review. J Quant Spectrosc Radiat Transfer 1996; 55: 535-575.

[3] Mishchenko MI, Travis LD, Macke A. T-matrix method and its applications. In Mishchenko MI, Hovenier JW, Travis LD, editors. Light scattering by nonspherical particles: theory, measurements, and applications. San Diego: Academic Press, 2000, p. 147-172. 
[4] Stammes P. Light scattering properties of aerosols and the radiation inside a planetary atmosphere. PhD thesis, Free University, Amsterdam, 1989.

[5] Kuik F, de Haan JF, Hovenier JW. Single scattering of light by circular cylinders. Appl Opt 1994; 33: 4609-4918.

[6] Mishchenko MI, Travis LD. Capabilities and limitations of a current FORTRAN implementation of the $T$-matrix method for randomly oriented, rotationally symmetric scatterers. J Quant Spectrosc Radiat Transfer 1998; 60: 309-324.

[7] Mishchenko MI, Travis LD, Macke A. Scattering of light by polydisperse, randomly oriented, finite circular cylinders. Appl Opt 1996; 35: 4927-4940.

[8] Mishchenko MI, Hovenier JW, Travis LD. Concepts, terms, notation. In Mishchenko MI, Hovenier JW, Travis LD, editors. Light scattering by nonspherical particles: theory, measurements, and applications. San Diego: Academic Press, 2000, p. 3-27.

[9] Hansen JE, Travis LD. Light scattering in planetary atmospheres. Space Sci Rev 1974; 16: $527-610$.

[10] Waldemarsson KWT, Gustafson BÅS. Light scattering by thin flakes. In Videen G, Fu Q, Chýlek P, editors. Light scattering by nonspherical particles: Halifax contributions. Adelphi, MD: US Army Research Laboratory, 2000, p. 34-37.

[11] Mishchenko MI, Hovenier JW. Depolarization of light backscattered by randomly oriented nonspherical particles. Opt Lett 1995; 20: 1356-1358.

[12] Sassen K. Lidar backscatter depolarization technique for cloud and aerosol research. In Mishchenko MI, Hovenier JW, Travis LD, editors. Light scattering by nonspherical particles: theory, measurements, and applications. San Diego: Academic Press, 2000, p. 393-416.

[13] Tozer WF, Beeson DE. Optical model of noctilucent clouds based on polarimetric measurements from two sounding rocket campaigns. J Geophys Res 1974; 79: 5607-5612.

[14] Witt G, Dye JE, Wilhelm N. Rocket-borne measurements of scattered sunlight in the mesosphere. J Atmos Terrestrial Phys 1976; 38: 223-238.

[15] West RA, Smith PH. Evidence for aggregate particles in the atmospheres of Titan and Jupiter. Icarus 1991; 90: 330-333.

[16] Tomasko MG, West RA, Castillo ND. Photometry and polarimetry of Jupiter at large phase angles. I. Analysis of imaging data of a prominent belt and a zone from Pioneer 10. Icarus 1978; 33: 558-592. 
[17] West RA, Lane AL, Hart H, Simmons KE, Hord CW, Coffeen DL, Esposito LW, Sato M, Pomphrey RB. Voyager 2 photopolarimeter observations of Titan. J Geophys Res 1983; 88: 8699-8707.

[18] Braak CF, de Haan JF, van der Mee CVM, Hovenier JW, Travis LD. Parameterized scattering matrices for nonspherical particles in planetary atmospheres. In Videen $G, F u Q$, Chýlek P, editors. Light scattering by nonspherical particles: Halifax contributions. Adelphi, MD: US Army Research Laboratory, 2000, p. 262-265.

[19] Weil H, Chu CM. Scattering and absorption of electromagnetic radiation by thin dielectric disks. Appl Opt 1976; 15: 1832-1836.

[20] Uzunoglu NK, Alexopoulos NG, Fikioris JG. Scattering from thin and finite dielectric fibers. J Opt Soc Am 1978; 68: 194-197.

[21] Schiffer R, Thielheim KO. Light scattering by dielectric needles and disks. J Appl Phys 1979; 50: 2476-2483.

[22] Weil H, Chu CM. Scattering and absorption by thin flat aerosols. Appl Opt 1980; 19: 20662071 .

\section{Figure captions}

Fig. 1. Phase function $a_{1}(\Theta)$ and ratio $a_{2}(\Theta) / a_{1}(\Theta)(\%)$ versus scattering angle $\Theta$ for spheres with size parameters ranging from 0.1 to 12 (see legend) and surface-equivalent, randomly oriented circular cylinders with diameter-to-length ratios $D / L=1$ and 20 . Note that for spheres $a_{2}(\Theta) / a_{1}(\Theta) \equiv 1$

Fig. 2. Ratios $a_{3}(\Theta) / a_{1}(\Theta)$ and $a_{4}(\Theta) / a_{1}(\Theta)(\%)$ versus scattering angle $\Theta$ for spheres with size parameters ranging from 0.1 to 12 and surface-equivalent, randomly oriented circular cylinders with diameter-to-length ratios $D / L=1$ and 20 . Note that for spheres $a_{3}(\Theta) \equiv a_{4}(\Theta)$.

Fig. 3. Ratios $-b_{1}(\Theta) / a_{1}(\Theta)$ and $b_{2}(\Theta) / a_{1}(\Theta)(\%)$ versus scattering angle $\Theta$ for spheres with size parameters ranging from 0.1 to 12 and surface-equivalent, randomly oriented circular cylinders with diameter-to-length ratios $D / L=1$ and 20 . 

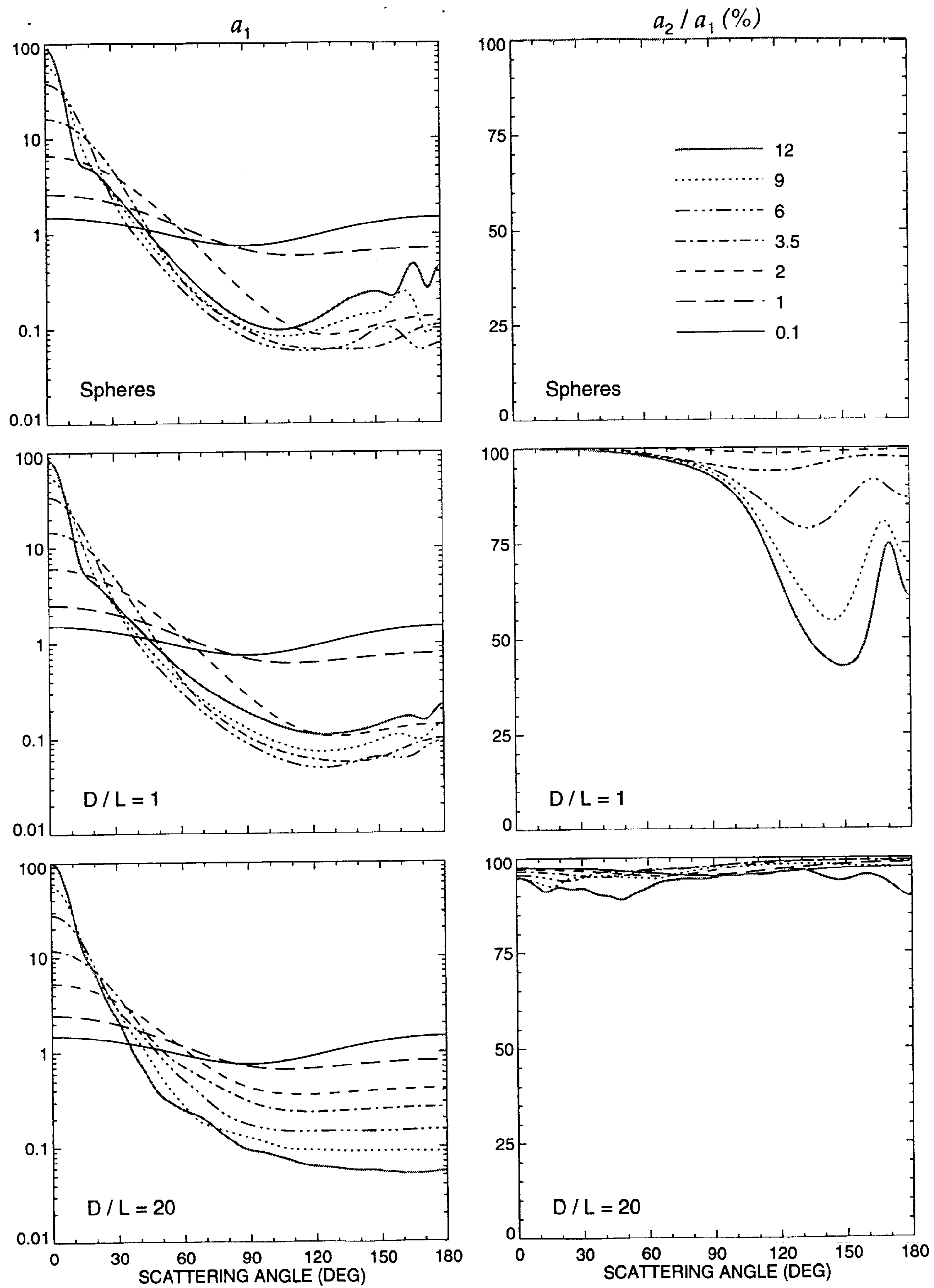

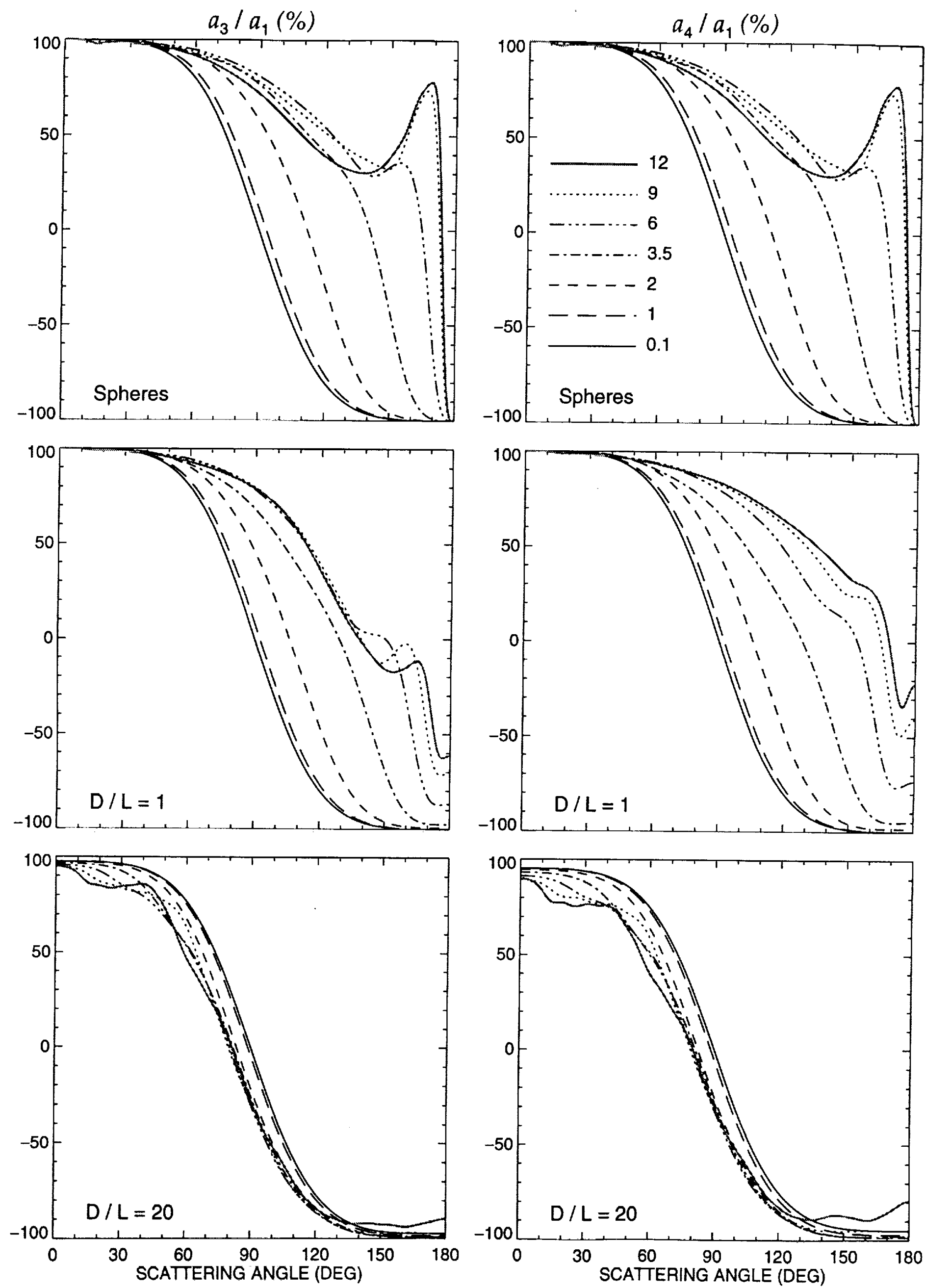

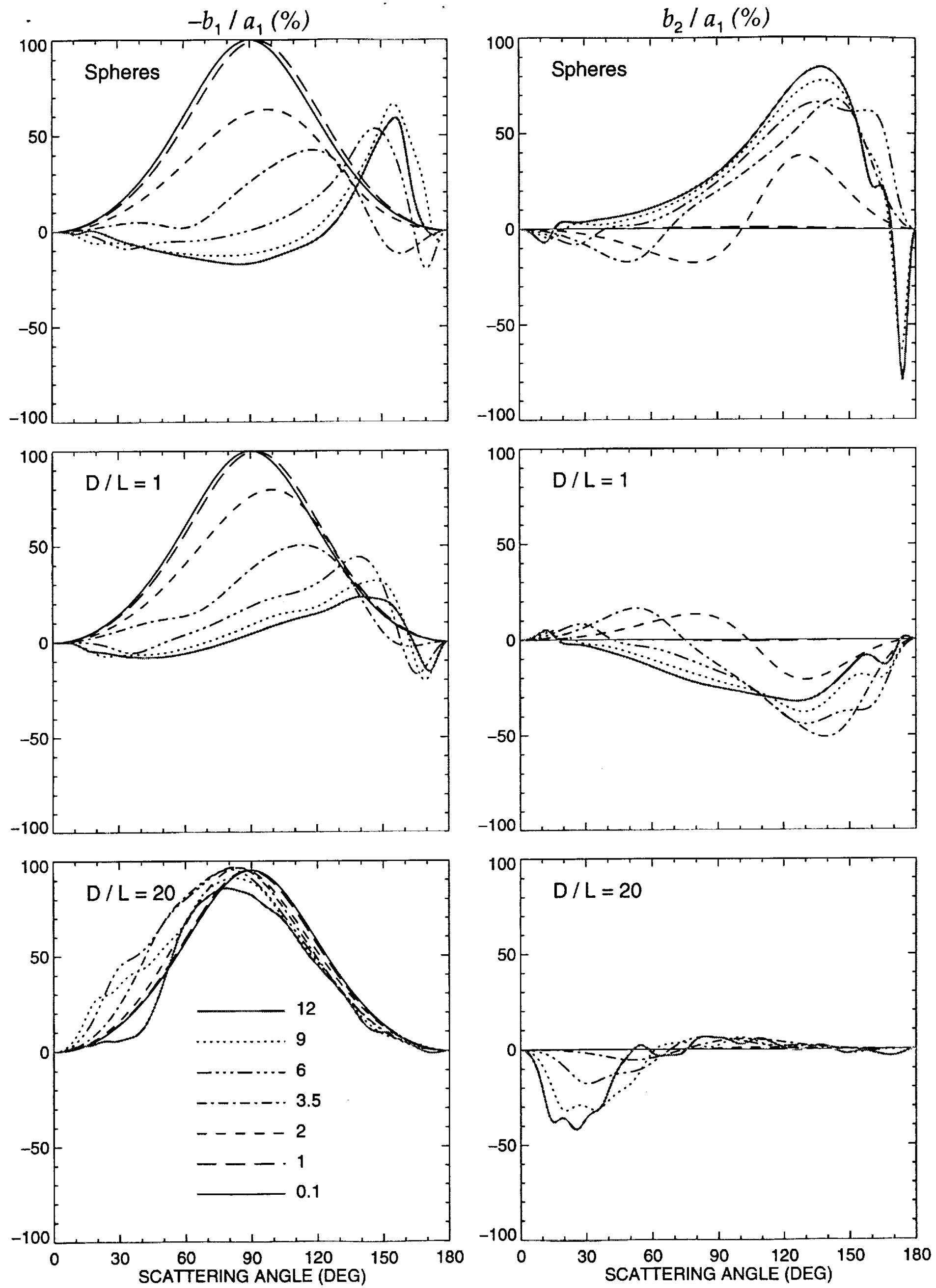
